UAB-FT-00-7

PLY-MS-00-7

\title{
Electrons and Photons: Fact not Fiction
}

\author{
EMILI BAGAN円 \\ Dept. Fisica Teòrica \& IFAE \\ Edifici Cn \\ Universitat Autònoma de Barcelona \\ E-08193 Bellaterra (Barcelona) \\ Spain \\ Martin LAVELlef and David MCMullan 3 \\ School of Mathematics and Statistics \\ The University of Plymouth \\ Plymouth, PL4 8AA \\ $U K$
}

\begin{abstract}
The particle Fock space of the matter fields in QED can be constructed using the free creation and annihilation operators. However, these particle operators are not, even at asymptotically large times, the modes of the matter fields that enter the QED Lagrangian. In this letter we construct the fields which do recover such particle modes at large times. We are thus able to demonstrate for the first time that, contrary to statements found in the literature, a relativistic description of charged particles in QED exists.
\end{abstract}

\footnotetext{
1email: bagan@ifae.es

${ }^{2}$ email: mlavelle@plymouth.ac.uk

3 email: dmcmullan@plymouth.ac.uk
} 


\section{Introduction}

The conclusions of this paper, that electrons are particles, will not surprise our experimental colleagues. What may come as a surprise to them is the fact that the hitherto accepted wisdom [1] 4 in the theoretical community was that there does not exist any relativistic description of the electron as a particle!

The root of the problem [5] lies in the masslessness of the photon. This generates long range interactions and it is well known that these fall off so slowly that they cannot be neglected even for widely separated charges a long time before or after scattering processes. This is seen in $S$-matrix calculations as the lack of a pole for fermionic external legs.

This lack of a particle interpretation of an electron presents a radical departure from the usual view of particle physics which we find highly unsatisfactory. We want to show that it is also unnecessary. We note that an understanding of how particles can arise in gauge theories is important to improve our insight into the physical structures of gauge theories, to help with QCD phenomenology and it may well have spin offs for the vexed question of how to describe unstable particles.

In the context of QED this lack of any particle language has not hindered progress. Our knowledge of the classical limit of QED acts as a guide to the extraction of physical predictions from suitably defined cross-sections via the Bloch-Nordsieck framework. In theories such as QCD such intuition is still greatly lacking. In particular we do not understand hadronisation which relies on coloured particles metamorphosing into jets. The route which leads from partons to effective quarks and glue is essentially uncharted.

In this letter we will show that a relativistic particle description of the electron (or any other charged particle) immediately follows once a correct physical identification of the electron has been made. Having first recalled the standard statement of the problem [1], we will show that the effects which prevent the identification of a charged particle structure in QED disappear if the right fields are used. We stress that this identification only holds in the asymptotic region a long time before or after scattering occurs. We then demonstrate that a particle structure is also asymptotically present for photons in full QED. Finally we discuss how these results have been verified in perturbative calculations and present some conclusions.

\section{The Interaction}

We have already noted that in theories like QED we have long range interactions due to

the masslessness of the photon. It has been shown by various authors that such interactions cannot be neglected even at large times before or after scattering. In particular, 
Kulish and Faddeev [1] showed that the annihilation operator of the matter fields of QED which is defined as the large time limit of the operator

$$
b(q, s, t):=\int d^{3} x \frac{1}{\sqrt{2 E_{q}}} u^{\dagger s}(q) \psi(x) \mathrm{e}^{i q \cdot x}
$$

does not become just the usual free particle mode, but rather takes on the formf

$$
b(q, s, t)=D_{\text {soft }}(q, t) b(q, s),
$$

where

$$
D_{\text {soft }}(q, t)=\exp \left\{-e \int_{\text {soft }} \frac{d^{3} k}{(2 \pi)^{3}} \frac{1}{2 \omega_{k}}\left(\frac{q \cdot a(k)}{q \cdot k} \mathrm{e}^{-i t k \cdot q / E_{q}}-\frac{q \cdot a^{\dagger}(k)}{q \cdot k} \mathrm{e}^{i t k \cdot q / E_{q}}\right)\right\},
$$

is called a distortion operator [1]. The creation and annihilation operators for the photonic variables enter into this expression and, as long as $D_{\text {soft }} \neq 1$, a particle mode for the electron will not be recovered. Of course, we should only expect to regain a particle description at asymptotic times and, for large $t$, the integral in (3) only receives contributions from soft photons, but it still does not reduce to the unit operator. This important observation has lead to the conclusion [1] that it is not possible to describe the electron as a particle.

However, since the interaction does not switch off, the matter field $\psi$ never becomes gauge invariant and thus we should not expect to identify it with physical particles via Equation ㅁ! We now construct the fields which do have a particle description at large times.

\section{The Electron}

We recall that physical fields must be invariant under the local gauge transformations, $A_{\mu}(x) \rightarrow A_{\mu}(x)+\partial_{\mu} \theta(x)$ and $\psi(x) \rightarrow \mathrm{e}^{i e \theta(x)} \psi(x)$. A physical matter field must then be given by a product of the form $h^{-1}(x) \psi(x)$ where, under a gauge transformation, $h^{-1}(x) \rightarrow h^{-1}(x) \mathrm{e}^{-i e \theta(x)}$. We call such a field with this gauge transformation property a dressing.

Of course there are a multiplicity of such dressing fields which satisfy this minimal requirement [6, []]. To describe a charged particle we need to further demand [8] that the dressing satisfies the dressing equation

$$
u \cdot \partial h^{-1}(x)=-i e h^{-1}(x) u \cdot A(x),
$$

\footnotetext{
${ }^{4}$ There is also a distortion contribution to the non-observable phase of $S$-matrix elements which we will ignore in this letter.
} 
where $u^{\mu}=\gamma(\eta+v)^{\mu}$ is the four velocity of the charged particle, $\eta$ is the unit time-like vector, $v=(0, \boldsymbol{v})$ is the velocity and $\gamma=\left(1-|\boldsymbol{v}|^{2}\right)^{-1 / 2}$. It is important to note the velocity dependence here: we can only expect to have a particle interpretation of a charge at asymptotic times and in that regime the velocity is a well defined quantum number. Our dressed charges will, therefore, only correspond to particles at the appropriate point on the mass shell characterised by the velocity in the dressing.

In QED we have been able to solve these two requirements [8,9] and have found the following description of a charged field moving with a given velocity:

$$
h^{-1}(x) \psi(x)=\mathrm{e}^{-i e K(x)} \mathrm{e}^{-i e \chi(x)} \psi(x) .
$$

The $K$ term is separately gauge invariant and contributes to the unobservable phase. We ignore it in this letter. The $\chi$ part of the dressing may be written as

$$
\chi(x)=\frac{\mathcal{G} \cdot A}{\mathcal{G} \cdot \partial},
$$

where $\mathcal{G}^{\mu}=(\eta+v)^{\mu}(\eta-v) \cdot \partial-\partial^{\mu}$.

We now define an annihilation operator for the gauge invariant, dressed field

$$
b(q, s, v, t):=\int d^{3} x \frac{1}{\sqrt{2 E_{q}}} u^{\dagger s}(q) \mathrm{e}^{-i e \chi(x)} \psi(x) \mathrm{e}^{i q \cdot x} .
$$

Note that this has an explicit velocity dependence coming from the form of the dressing. To find its asymptotic form we proceed in the same manner as Kulish and Faddeev [1]. The annihilation operator now picks up two distortion factors: the original one associated with the unphysical matter field and a further correction from the dressing. We find

$$
b(q, s, t, v) \rightarrow h_{\mathrm{soft}}^{-1}(q, t, v) D_{\mathrm{soft}}(q, t) b(q, s),
$$

where $D_{\text {soft }}(q, t)$ is given in (3). The distortion from the soft part of the dressing is

$$
h_{\text {soft }}^{-1}(q, t, v)=\exp \left\{e \int_{\text {soft }} \frac{d^{3} k}{(2 \pi)^{3}} \frac{1}{2 \omega_{k}}\left(\frac{V \cdot a(k)}{V \cdot k} \mathrm{e}^{-i t k \cdot q / E_{q}}-\frac{V \cdot a^{\dagger}(k)}{V \cdot k} \mathrm{e}^{i t k \cdot q / E_{q}}\right)\right\}
$$

where $V^{\mu}=(\eta+v)^{\mu}(\eta-v) \cdot k-k^{\mu}$, which is essentially the Fourier transform of $\mathcal{G}^{\mu}$. Combining these distortions we find the overall distortion factor

$$
\begin{aligned}
h_{\text {soft }}^{-1}(q, t, v) D_{\text {soft }}(q, t)=\exp \left(e \int_{\text {soft }} \frac{d^{3} k}{(2 \pi)^{3}}\right. & \frac{1}{2 \omega_{k}}\left[\left(\frac{V \cdot a(k)}{V \cdot k}-\frac{q \cdot a(k)}{q \cdot k}\right) \mathrm{e}^{-i t k \cdot q / E_{q}}\right. \\
& \left.\left.-\left(\frac{V \cdot a^{\dagger}(k)}{V \cdot k}-\frac{q \cdot a^{\dagger}(k)}{q \cdot k}\right) \mathrm{e}^{i t k \cdot q / E_{q}}\right]\right) .
\end{aligned}
$$


We now note that we can write (recall that $k$ is on-shell)

$$
\begin{aligned}
\frac{V^{\mu}}{V \cdot k}-\frac{q^{\mu}}{q \cdot k} & =\frac{(\eta+v)^{\mu}(\eta-v) \cdot k-k^{\mu}}{(\eta+v) \cdot k(\eta-v) \cdot k}-\frac{q^{\mu}}{q \cdot k} \\
& =\frac{(\eta+v)^{\mu}}{(\eta+v) \cdot k}-\frac{q^{\mu}}{q \cdot k}-\frac{k^{\mu}}{V \cdot k} .
\end{aligned}
$$

Hence, at the point in the mass-shell where $q^{\mu}=m \gamma(\eta+v)^{\mu}$, the distortion operator becomes a trivial operator since the argument of the exponential becomes

$$
-e \int \frac{d^{3} k}{(2 \pi)^{3}} \frac{1}{2 \omega_{k}}\left(\frac{k \cdot a(k)}{V \cdot k} \mathrm{e}^{-i t \omega_{k}}-\frac{k \cdot a^{\dagger}(k)}{V \cdot k} \mathrm{e}^{i t \omega_{k}}\right)
$$

which vanishes between physical states due to the Gupta-Bleuler subsidiary condition (it only contains unphysical photon degrees of freedom which correspond to the NakanishiLautrup $B$ field). We thus see that the modes of the dressed field, at the appropriate place in the mass shell, are free particle modes at large times. There is no distortion and we have recovered a particle picture for charged matter in QED.

\section{The Photon}

In the usual framework it is assumed that the coupling switches off asymptotically. There are then many ways to show that the physical components of the non-interacting vector potential are the transverse degrees of freedom (see, e.g., Chap. 19 of [10]). However, we have seen that in the true asymptotic domain the coupling does not vanish due to the masslessness of the photon. This means that previous arguments are incomplete. We will now show that, although we still have an interaction, the physical photonic degrees of freedom do decouple and a particle description for the photon can be recovered in full QED.

The asymptotic form of the (interaction picture) vector boson can be readily obtained once the form of the asymptotic interaction Hamiltonian, $H_{\mathrm{int}}^{\mathrm{as}}$, has been identified [1, 11]. Using this result we can straightforwardly transform from the free vector boson, $A^{\mathrm{f}}$, to the asymptotic Heisenberg field, $A^{\text {as }}$, as follows:

$$
\begin{aligned}
A_{\mu}^{\mathrm{as}}(x) & =\exp \left(i \int_{-\infty}^{t} d \tau H_{\mathrm{int}}^{\mathrm{as}}(\tau)\right) A_{\mu}^{\mathrm{f}}(x) \exp \left(-i \int_{-\infty}^{t} d \tau H_{\mathrm{int}}^{\mathrm{as}}(\tau)\right) \\
& =A_{\mu}^{\mathrm{f}}(x)-e \int_{-\infty}^{t} d \tau d^{3} y D(\tau-t, \boldsymbol{y}-\boldsymbol{x}) J_{\mu}^{\mathrm{as}}(\tau, \boldsymbol{y}),
\end{aligned}
$$

where the asymptotic current is given by

$$
J_{\mathrm{as}}^{\mu}(t, \boldsymbol{x})=\int \frac{d^{3} p}{(2 \pi)^{3}} \frac{p^{\mu}}{E_{p}} \rho(p) \delta^{3}\left(\boldsymbol{x}-t \boldsymbol{p} / E_{p}\right) .
$$


This shows, as expected, that the vector boson field is also not free at large times. In the spirit of our discussions above, we can, though, straightforwardly recover a particle picture for photons by showing that the second term in (13) vanishes for the transverse, physical components, $\left(\delta_{i j}-\partial_{i} \partial_{j} / \nabla^{2}\right) A_{j}^{\text {as }}$. This shows that in the far field domain, where the potential takes on the asymptotic form (13), a particle description emerges even in the interacting theory.

\section{Conclusions}

In this letter we have shown how a particle description for the electron and the photon emerges from QED. The essential observation in this construction is that a particle cannot be identified with the raw matter field that enters into the QED Lagrangian. Rather, a physical particle corresponds to an appropriately dressed, gauge invariant field. The dressing for a charged asymptotic particle with a specific velocity depends explicitly on that velocity. A particle description for the electron is only recovered at that point in the mass shell corresponding to the velocity.

For a photon it is not surprising that not all the components of the vector potential are physical. What is new in our discussion of the photon is that we have seen how the free photon emerges in the asymptotic regime even though there is still a residual interaction with matter for the non-physical components.

The formal arguments presented in this letter have been checked in a wide variety of detailed calculations. We have shown 112, 13 for both scalar and fermionic QED that the on-shell propagator and other Green's functions of the dressed matter fields have, to all orders in perturbation theory, a good pole structure. This is in contrast to the Lagrangian matter fields whose on-shell Green's functions are plagued by infra-red singularities. This means [9] that for our fields the $S$-matrix elements can be constructed via the traditional LSZ-formalism.

We should also point out that these charged fields have good ultra-violet behaviour [13, 14]. Multiplicative renormalisation is possible and the dressed field operators do not mix under renormalisation [13]. In addition, there is an ultra-violet logarithm in scattering processes [13] which is just the universal Isgur-Wise (or equivalently the Wilson line

kink) renormalisation constant. This logarithm structure is just the one that appears in the Bloch-Nordsieck formalism.

This programme is currently being extended to the non-abelian domain where the theoretical problems are more severe [15, 16] and the experimental situation in identifying physical quarks and gluons, e.g., in jets, is much more subtle. The essential new ingredient found there that further obstructs a particle interpretation is the gluonic selfinteraction. Such massless charges spawn a new class of asymptotic dynamics which is no longer spin independent and generates collinear singularities. This can be studied 
in massless QED and early indications [17] are that the appropriate dressed massless charges are indeed free. Furthermore, we have already seen 18 that the minimal, nonabelian component of the dressing is responsible for the anti-screening interaction that drives asymptotic freedom in both three and four dimensional QCD. These results give us a great deal of confidence that we can regain a particle picture of quarks and gluons in the pre-hadronisation regime.

Acknowledgements: This work was supported by the British Council/Spanish Education Ministry Acciones Integradas grant no. 1801/HB1997-0141. We thank Robin Horan, Tom Steele, Shogo Tanimura and Izumi Tsutsui for discussions.

\section{References}

[1] P. P. Kulish and L. D. Faddeev, Theor. Math. Phys. 4, 745 (1970).

[2] D. Zwanziger, Phys. Rev. D14, 2570 (1976).

[3] J. Frohlich, G. Morchio, and F. Strocchi, Ann. Phys. 119, 241 (1979).

[4] D. Buchholz, M. Porrmann, and U. Stein, Phys. Lett. B267, 377 (1991).

[5] J. Dollard, J. Math. Phys. 5, 729 (1964).

[6] P. A. M. Dirac, Can. J. Phys. 33, 650 (1955).

[7] M. Lavelle and D. McMullan, Phys. Rept. 279, 1 (1997), hep-ph/9509344.

[8] E. Bagan, M. Lavelle, and D. McMullan, Phys. Rev. D57, 4521 (1998), hepth/9712080.

[9] E. Bagan, M. Lavelle, and D. McMullan, (1999), hep-ph/9909257, Charges from Dressed Matter: Construction, submitted for publication.

[10] M. Henneaux and C. Teitelboim, Quantization of Gauge Systems, Princeton University Press, 1992.

[11] R. Horan, M. Lavelle, and D. McMullan, (1999), hep-th/9909044, Asymptotic Dynamics in Quantum Field Theory, submitted for publication.

[12] E. Bagan, M. Lavelle, and D. McMullan, Phys. Rev. D57, 4521 (1998), hepth/9712080. 
[13] E. Bagan, M. Lavelle, and D. McMullan, (1999), hep-ph/9909262, Charges from Dressed Matter: Physics and Renormalisation, submitted for publication.

[14] E. Bagan, M. Lavelle, and D. McMullan, Phys. Rev. D56, 3732 (1997), hepth/9602083.

[15] M. Ciafaloni, in Quantum Chromodynamics, Ed. A.H. Mueller (World Scientific, Singapore 1989).

[16] D. Buchholz, Nucl. Phys. B469, 333 (1996), hep-th/9511002.

[17] R. Horan, M. Lavelle, and D. McMullan, Pramana J. Phys. 51, 317 (1998), hepth/9810089, Erratum-ibid, 51 (1998) 235.

[18] M. Lavelle and D. McMullan, Phys. Lett. B436, 339 (1998), hep-th/9805013. 